\title{
Phosphate Solubilizing Microorganisms for Cereal Production in Adamawa (Cameroon)
}

\author{
A. Maimouna, T. L. Tchuenteu, D. Nwaga, J. P. Nguetnkam, and C. Megueni
}

\section{ABSTRACT}

Phosphorus is usually a limiting factor of acidic tropical soil and limited by the low availability and high cost. The present study aimed to evaluate the interaction of Hangloa vivianite (as phosphorus supply), the selected Mycorrhizal fungi strains (M) and Phosphate Solubilizing Microorganisms (PSM) on maize production in the Adamawa Region of Cameroon. An experimental design with 15 combinations of bacteria strains (B), ( BGL12 as B1, SSL9 as B2, BNBL17 as B3, RBNBL5 as B4, and the combinations of these singles strains, namely B1B2, B1B3, B1B4, B2B3, B2B4, B3B4, B1B2B3, B1B2B4, B1B3B4, B2B3B4, B1B2B3B4) and 05 inputs (mycorrhiza (M), Rock Phosphate (RP) and mixture of M with RP $(\mathrm{RP}+\mathrm{M}))$, negative control $(\mathrm{C}$-) and positive control $(\mathrm{C}+))$ was used. After four months of growth, nutrients (Phosphorus and Nitrogen) content and grain yield were assessed. Results indicated that total phosphorus content of M, RP and RP+M plants were 2.42, 2.11 and 4.00 fold higher than that of unfertilized plants. M, RP and RP+M increased maize seeds yield by $26.20 \%, 26.89 \%$ and $165.51 \%$ relative to negative control. The benefit of the selected PSM with Mycorrhizal fungi strains (246.76\% for N; 216.34\% for $P$ ) and with the Rock Phosphate $(314.23 \%$ for $N$; $167.26 \%$ for $P$ ) was more pronounced with the combination of $R P+M(1030.45 \%$ for $N$ and 967.31\% for P). These results showed that PSM associated with Mycorrhiza can be used as inoculants to improve the efficiency of vivianite as phosphate fertilizer for sustainable maize production under SudanoGuinean Climate of Adamawa Cameroon region.

Keywords: Grain yield, Hangloa vivianite, Adamawa Cameroon, Phosphates Solubilizing Microorganisms, Phosphorus uptake.

\section{INTRODUCTION}

Cereals are species generally sown for their grains, whose starchy albumen, reduced in flour, is eaten by human or by domestic animals. Most cereals belong to the Poaceae family. These are: wheat, rice, millet, sorghum, barley, oats, rye, corn. The main cereals produced in the world, is Maize (Zea mays L.) with approximately 900 million tons produced per year [32]. Zea mays L. is an important food crop in sub-Saharan Africa (SSA) that contributes significantly to food security [3]. In Northern Cameroon, where Maize is the most important cereal food, the quantities produced are not enough for this Region. Soil fertility is among the major factors affecting maize production in this Region. Arbuscular Mycorrhizal Fungi (AMF) present in soils, forming associations with roots provide phosphate $(\mathrm{P})$ and other nutrients to their plant hosts by producing hyphae that grow out from roots [31]. However, AMF can only exploit soluble $\mathrm{P}$ sources and much $\mathrm{P}$ in the soil is in an insoluble form. To increase the production of some cereals, chemical fertilizers such as phosphorus were used, but their high cost limited their availability for farmers. Phosphorus (P) is an essential nutrient for plant such as maize and plays many roles in nutrient uptake, in respiration, biological oxidation, photosynthesis and this $\mathrm{P}$ is a structural component of
Published Online: November 28, 2020

ISSN: 2684-5199

DOI :10.24018/ejbio.2020.1.6.102

\section{A. Maimouna *}

University of Ngaoundere, Cameroon. (e-mail: abbamouna@gmail.com)

T. L. Tchuenteu

University of Ngaoundere, Cameroon.

(e-mail: tatchumlucien@yahoo.fr)

D. Nwaga

University of Yaounde, Cameroon. (e-mail: dnwaga@yahoo.fr)

J. P. Nguetnkam

University of Ngaoundere, Cameroon.

(e-mail: jpnguet@yahoo.fr)

C. Megueni

University of Ngaoundere, Cameroon.

(e-mail: cmegueni2003@yahoo.fr)

*Corresponding Author nucleic acid [9]. It is also important in the process of the flowering and grain filling stages [33]. To ensure the plant production, millions of tons of $\mathrm{P}$ fertilizer were added to soils each year [14]. The use of these $P$ chemicals fertilizers has increased crop yields, but their excessive and abusive use has resulted in human health pollution and environment pollution [28]. In this respect, the use of many natural fertilizers (Rock Phosphate Powder or Rock Potassium Powder) and biofertilizers is an alternative. Rock Phosphate are sources of $\mathrm{P}$ but not readily available to a plant because this mineral is released slowly and their use as fertilizer often causes insignificant yield increases of crop production [36]. Microorganisms have the ability to solubilize the insoluble phosphates and maintain the nutrient status of soil [27]. The use of Plant Growth Promoting Rhizobacteria (PGPR) and Phosphorus Solubilizing Microorganisms (PSM) as biofertilizers, contribute to improve plant growth, plant nutrition and agricultural production [7], [22], [24], [29], [19]. PSM are known for their ability to solubilize inorganic phosphates, making phosphorus available to plants [10], [8], [16]. Unfortunately, cereal production decreased in Sudano-Sahelian and Guinea-savannah zones during this last decade, due to the decrease of soil fertility. In the region of Adamawa (Cameroon) where soils are ferralitic, this loss of fertility is mainly due to high soil acidity and nutrient leaching by rainfall [20]. In this region, 
there are several geological materials rich in $\mathrm{P}$ and other mineral element that can be used to amend soils and improve crop production. Hangloa vivianite is one of the local geological materials rich in phosphate and accessible to peasants [35]. Our study focused on improvement of cereal production in the Sudano-Guinean zones of Cameroon. In this respect Phosphate Solubilizing Microorganism (PSM) was isolated from Sorghum (Sorghum bicolor L. Moench) and maize (Zea mays L.) rhizosphere. Results indicate that the selected bacteria stimulated maize growth in pots and in fields. Also, PSM have shown their potential to improve by 50 to $160 \%$ Phosphorus absorption by Sorghum bicolor and Vigna unguiculata under greenhouse conditions [15]. The author [7] Reported that inoculation of Phosphate Solubilizing Bacteria ( $P$. fluorescens), isolated from acid soils in Cameroon and able to mobilize $\mathrm{P}$ from $\mathrm{Al}-$ and $\mathrm{Fe}-$ phosphates, increased grain yield and $\mathrm{P}$ uptake of maize when tested in the greenhouse. The main objective of this study was to improve durably cereal production in the Northern Cameroon. Therefore, PSM isolates, Rock Phosphate and Mycorrhizae were used to improve grain yield, nitrogen uptake and phosphorus-solubilization. The interest and usefulness of this work was that the combination of PSM, vivianite powder and mycorrhizae which better improve maize productivity will be popularized.

\section{A. Maize Seeds}

\section{MATERIAL}

Shaba variety of maize obtained from the Regional Delegation of MINADER was used (Fig. 1). It is an improved variety with a four-month life cycle. This variety of maize is cultivated by Adamawa's farmers because of its good quality of flour used as food.

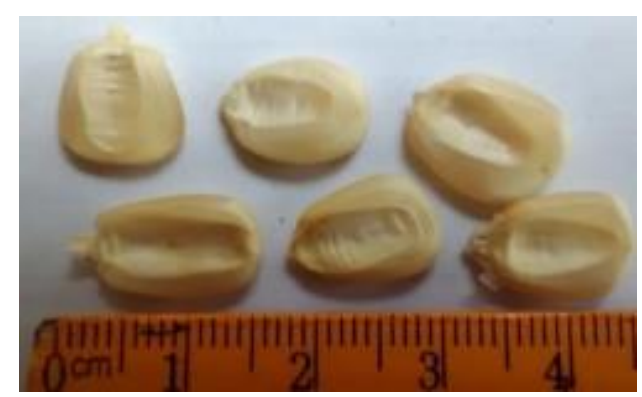

Fig. 1. Seeds of Zea mays Var Shaba.

\section{B. Biofertilizers}

Phosphates Solubilizing Microorganisms (PSM) and selected Mycorrhizal fungi strains (M) were used as biofertilizers. Four single selected strains of PSM (BGL1 as B1, SSL9 as B2, BNBL17 as B3 and RBNBL5 as B4) isolated from maize and sorghum rhizosphere and 11 combinations of these strains were used in these experiments.

The Mycorrhizal biofertilizers (Fig. 2) used were supply by the Laboratory of Microbiology and Soils of the Biotechnology Center of the University of Yaounde I. Selected isolates were multiplied on sorghum and peanut plants (hosts plants) and tested on maize and sorghum [23]. It contains infective spores and propagules of three genera: Glomus, Scutellospora and Gigaspora species with a concentration of 20 spores/g of substrate.

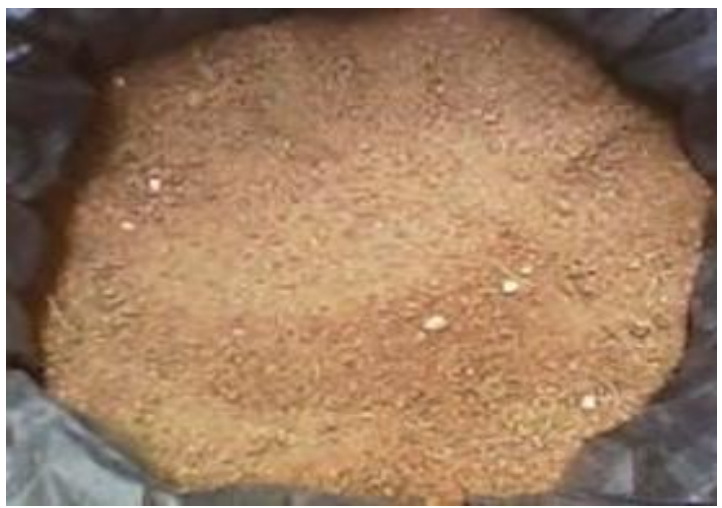

Fig. 2. Inoculum of mycorrhizal fungi.

\section{Rock Phosphate}

A Rock Phosphate used was obtained from vivianite (Fig. 3). It was sampled from the Hangloa Basin located at about $25 \mathrm{~km}$ in the Northwest of Ngaoundere city. Chemical analyses of this powder were done by [35] and were given the following composition: $\mathrm{Fe}_{2} \mathrm{O}_{3}$ (68.72\%), $\mathrm{P}_{2} \mathrm{O}_{5}(9.17 \%), \mathrm{Al}_{2} \mathrm{O}_{3}(7.72 \%)$ and $\mathrm{SiO}_{2}$ (9.67\%), total Phosphorus $(671.50 \mathrm{mg} / \mathrm{kg})$ and available phosphorus $(81.13 \mathrm{mg} / \mathrm{kg})$.
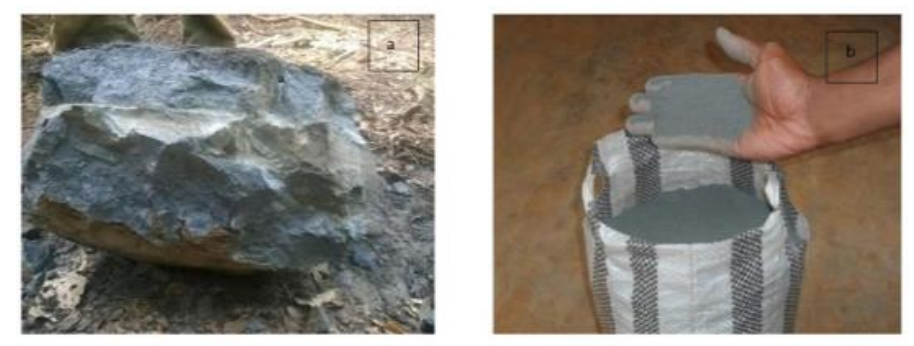

Fig. 3. a) Vivianite and b) Rock powder of vivianite.

\section{METHODS}

A. Effects of Phosphate Solubilizing Microorganisms (PSM), Mycorrhizal fungi and Rock Phosphate on Maize Growth in Field.

A $15 \times 5$ experimental design (Table I) with 15 combinations of bacteria (B), (BGL12 as B1, SSL9 as B2, BNBL17 as B3, RBNBL5 as B4, and the combinations of these singles strains, namely B1B2, B1B3, B1B4, B2B3, $\mathrm{B} 2 \mathrm{~B} 4, \mathrm{~B} 3 \mathrm{~B} 4, \mathrm{~B} 1 \mathrm{~B} 2 \mathrm{~B} 3, \mathrm{~B} 1 \mathrm{~B} 2 \mathrm{~B} 4, \mathrm{~B} 1 \mathrm{~B} 3 \mathrm{~B} 4, \mathrm{~B} 2 \mathrm{~B} 3 \mathrm{~B} 4$, $\mathrm{B} 1 \mathrm{~B} 2 \mathrm{~B} 3 \mathrm{~B} 4)$ and 05 inputs [combinations of mixture of Rock Phosphate with the selected Mycorrhizal fungi strains (mycorrhizae (M), Rock Phosphate (RP) and mixture of M with RP (RP+M)) negative control (C-) and positive control $(\mathrm{C}+)$ ] was used. The elementary plots were installed in 3 randomized blocks. Each block had the following measure: $32.25 \mathrm{~m} \times 30.5 \mathrm{~m}$. The dimension of one elementary plot was $3.75 \mathrm{~m} \mathrm{x} 3.5 \mathrm{~m}$ (area $\left.=13.125 \mathrm{~m}^{2}\right)$ separated from another by 1 meter. There were 145 elementary plots (15 isolates and combination of isolates plus positive and negative control) replicated three times. The space between the lines was $75 \mathrm{~cm}$ and $50 \mathrm{~cm}$ in line. The quantity of 
inputs was $15 \mathrm{~g}$ of $\mathrm{M}, 50 \mathrm{~g}$ of RP and mixture of $15 \mathrm{~g}$ of $\mathrm{M}$ with $50 \mathrm{~g}$ of RP were applied per hole.

Maize crops were grown during the rainy cropping season in the Adamawa Region. Maize seeds were disinfected alternately in $70 \%$ ethanol for $1 \mathrm{~min}$ and in sodium hypochlorite $5 \%$ during $5 \mathrm{~min}$ then rinsed several times with sterile water. These sterilized seeds were pregerminated in a Petri dish with sterile distilled water. Two pregerminated seeds were introduced in each hole. Inoculation of PSM was carried out before sowing by coating of pregerminated seeds maize. Mycorrhizal fungi were inoculated at a rate of $15 \mathrm{~g}$ per hole. Likewise, the Rock Phosphate was applied in the front hole planted at 50 $\mathrm{g}$ per hole before sowing.

\begin{tabular}{ll}
\multicolumn{2}{c}{ TABLE I: CODES OF PSM ISOLATES AND THEIR COMBINATION } \\
\hline Codes & Isolates \\
\hline B1 & BGL12 \\
B2 & SSL9 \\
B3 & BNBL17 \\
B4 & RBNBL5 \\
B1B2 & BGL12+SSL9 \\
B1B3 & BGL12+BNBL17 \\
B1B4 & BGL12+RBNBL5 \\
B2B3 & SSL9+BNBL17 \\
B2B4 & SSL9+RBNBL5 \\
B3B4 & BNBL17+RBNBL5 \\
B1B2B3 & BGL12+SSL9+BNBL17 \\
B1B2B4 & BGL12+SSL9+RBNBL5 \\
B1B3B4 & BGL12+BNBL17+RBNBL5 \\
B2B3B4 & SSL9+BNBL17+RBNBL5 \\
B1B2B3B4 & BGL12+SSL9+BNBL17+RBNBL5 \\
\hline
\end{tabular}

\section{B. Nitrogen and Phosphorus contents}

Nitrogen and Phosphorus analysis was undertaken at the Laboratory of Microbiology and Soils at the University of Yaoundé I. The colorimetric methods described by [21] for phosphorus and [5] for nitrogen content determination were used. Dried plants were crushed for those analyses and yield parameters were undertaken.

\section{Statistical Analysis}

The data were statistically analyzed with "XLSTAT 2017 and EXCEL 2013" software. Analysis of variance (ANOVA) allowed the variability of dependent data to be assessed. Means were separated by Duncan's test.

\section{A. Weight of Maize Cobs}

\section{RESULTS}

After four months of growth, a highly significant difference $(\mathrm{P}<0.001)$ was observed between the effects of PSM isolates on the average weight of maize cobs for the $\mathrm{RP}+\mathrm{M}$ treatment in general (Fig. 4). The Rock Phosphate associated with PSM improved the weight of the maize cobs for B3, B4, B1B2, B1B3, B1B4, B3B4, B2B3B4 and $\mathrm{B} 1 \mathrm{~B} 2 \mathrm{~B} 3 \mathrm{~B} 4$ isolates. Elsewhere, with mycorrhizae, all PSM isolates alone or in combination, significantly increased highly the weight of maize cobs $(\mathrm{P}<0.01)$. The highest values were obtained with the combination of isolates B2B3B4 (267.06 $\pm 0.76 \mathrm{~g})$, B2B3B4 (308.76 $\pm 4.05 \mathrm{~g})$ and $\mathrm{B} 1 \mathrm{~B} 2(512.33 \pm 1.79 \mathrm{~g})$ in treatments $\mathrm{M}, \mathrm{RP}$ and $\mathrm{RP}+\mathrm{M}$ respectively; In the $\mathrm{RP}+\mathrm{M}$ treatment, analysis of variance showed that all isolates were significantly different to the negative control (C-).

\section{B. Plant Nitrogen Content}

Fig. 5 showed nitrogen content in maize plant. It ranges from $1.97 \pm 0.60 \mathrm{mg} / 100 \mathrm{~g}$ (C-) to $5.13 \pm 0.14 \mathrm{mg} / 100 \mathrm{~g}$ (B1) for M treatment, from $2.44 \pm 0.26 \mathrm{mg} / 100 \mathrm{~g}$ (B1B3B4) to $5.57 \pm 0.308 \mathrm{mg} / 100 \mathrm{~g}(\mathrm{~B} 2 \mathrm{~B} 3 \mathrm{~B} 4)$, for RP treatment and from $1.97 \pm 0.60 \mathrm{mg} / 100 \mathrm{~g}$ (C-) to $6.85 \pm 0.15 \mathrm{mg} / 100 \mathrm{~g}$ of dry matter (B2B3B4) for the RP+M treatment. All PSM isolates have significantly improved $(\mathrm{P}<0.05)$ the plants nitrogen content in the three treatments when compared with positive control. It appears that there was a significant difference between treatments $\mathrm{M}$ and $\mathrm{RP}+\mathrm{M}$ and between treatments $\mathrm{RP}$ and $\mathrm{RP}+\mathrm{M}$. In the $\mathrm{RP}+\mathrm{M}$ treatment, PSM improve nitrogen absorption. The interaction between treatments and isolates was highly significant $(\mathrm{P}<0.001)$.

\section{Plant Phosphorus Content}

The highest value was obtained with isolates B1B3 $(0.30 \pm 0.02 \mathrm{mg} / 100 \mathrm{~g}), \mathrm{B} 1 \mathrm{~B} 3 \mathrm{~B} 4(0.42 \pm 0.01 \mathrm{mg} / 100 \mathrm{~g})$ and B2B3B4 $(0.60 \pm 0.03 \mathrm{mg} / 100 \mathrm{~g})$ in treatments $\mathrm{M}, \mathrm{RP}$ and $\mathrm{RP}+\mathrm{M}$ respectively (Fig. 6). The lowest values were obtained by the $\mathrm{C}+(0.05 \pm 0.01 \mathrm{mg} / 100 \mathrm{~g})$ and the $\mathrm{C}$ $(0.09 \pm 0.02 \mathrm{mg} / 100 \mathrm{~g})$. As with nitrogen, mycorrhizae alone or associated with RP improved the plant phosphorus content.

\section{Maize Field Yield}

The positive effect of PSM inoculations with mycorrhizae and Rock Phosphate were clearly observed in Table II and III. The highest straw yield is observed with the associated isolate B1B2 $(5.71 \pm 0.00 \mathrm{t} / \mathrm{h})$ and the minimum is observed with B1B3B4 $(2.63 \pm 0.01 \mathrm{t} / \mathrm{h})$ in the treatment $\mathrm{RP}+\mathrm{M}$. The maximum in the isolated RP and M treatments were observed with association of isolates B2B3B4 $(2.83 \pm 0.00 \mathrm{t} / \mathrm{h})$, B1B3B4 $(2.57 \pm 0.01 \mathrm{t} / \mathrm{h})$ while the minimums were observed with association of isolates B1B3B54 $(1.18 \pm 0.01 \mathrm{t} / \mathrm{h})$ and B1B3 $(1.40 \pm 0.01 \mathrm{t} / \mathrm{h})$, respectively.

All isolates significantly ( $\mathrm{P}<0.001)$ improved the yield of maize in the three treatments with the exception of isolates B4 and B1B2 which did not show any significance compared to the negative control in the RP treatment.

\section{E. Total Nitrogen Yield and Response to Total Nitrogen Uptake by Maize Plants}

The highest total nitrogen yield was obtained with isolates B1B3B4 $(202.89 \pm 8.10 \mathrm{Kg} / \mathrm{ha}), \mathrm{B} 2 \mathrm{~B} 3 \mathrm{~B} 4(321.30 \pm$ $3.20 \mathrm{Kg} / \mathrm{ha})$, and B1B2 $(570.7 \pm 1.98 \mathrm{Kg} / \mathrm{ha})$ in treatments $\mathrm{M}, \mathrm{RP}$ and $\mathrm{RP}+\mathrm{M}$ respectively (Table II) and the smallest percentage was rated with the negative control $(0.00 \pm 0.00 \%)$. Analysis of variance showed that a significant difference was observed between the effects of isolates and the mean yield content and nitrogen absorption for the $\mathrm{RP}+\mathrm{M}$ treatment $(\mathrm{P}<0.001)$.

\section{F. Total Phosphorus Yield and Response to Total Phosphorus Uptake by Maize Plants}

The results reported in Table III showed that the highest values were obtained with isolates B1B3B4 $(15.70 \pm 0.01 \mathrm{Kg} / \mathrm{ha}), \quad$ B2B3B4 $(9.60 \pm 1.62 \mathrm{Kg} / \mathrm{ha}), \quad$ and 
B2B3B4 $(41.04 \pm 1.23 \mathrm{Kg} / \mathrm{ha})$ in treatments $\mathrm{M}, \mathrm{RP}$ and $\mathrm{RP}+\mathrm{M}$, respectively. The lowest value was obtained with the negative control $(1.29 \pm 0.00 \mathrm{Kg} / \mathrm{ha})$ in the three treatments. The highest percentage was rated by the association of isolates B1B3B4 (5638.21 $\pm 1.80 \%)$, B1B3 $(301.21 \pm 1.22 \%)$ and B2B3B4 $(1568.29 \pm 1.74 \%)$ in treatments $\mathrm{M}, \mathrm{RP}$ and $\mathrm{RP}+\mathrm{M}$, respectively. The analysis of variance showed that a significant difference is observed between the effects of isolates and the mean yield content and absorption of phosphorus for $\mathrm{RP}+\mathrm{M}$ treatment $(\mathrm{P}<0.001)$.
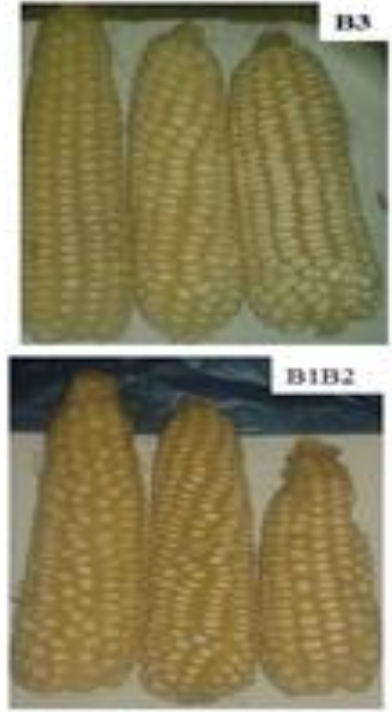

M
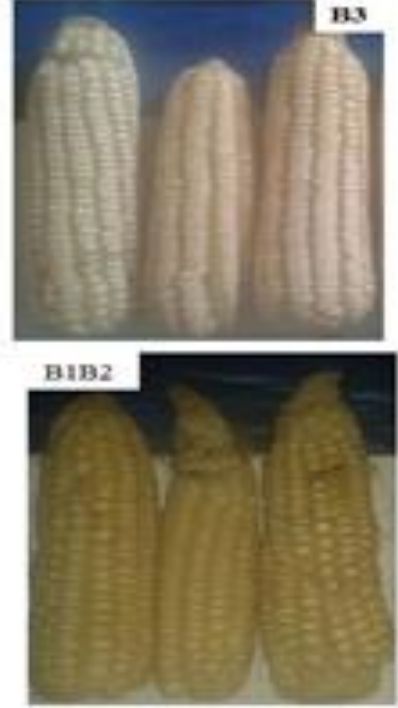

RP
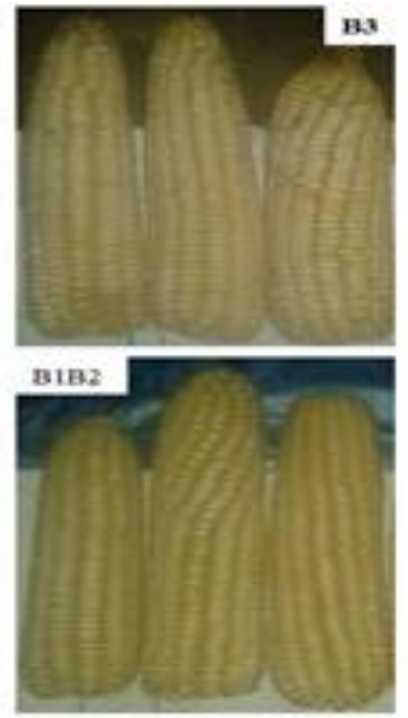

$\mathrm{RP}+\mathrm{M}$

Fig. 4. Maize cobs harvested in fields.

Legend: B3: SSL9; B1B2: BGL12+SSL9; RP: Rocks Phosphates; RP+M: Rocks Phosphates and Mycorrhiza.

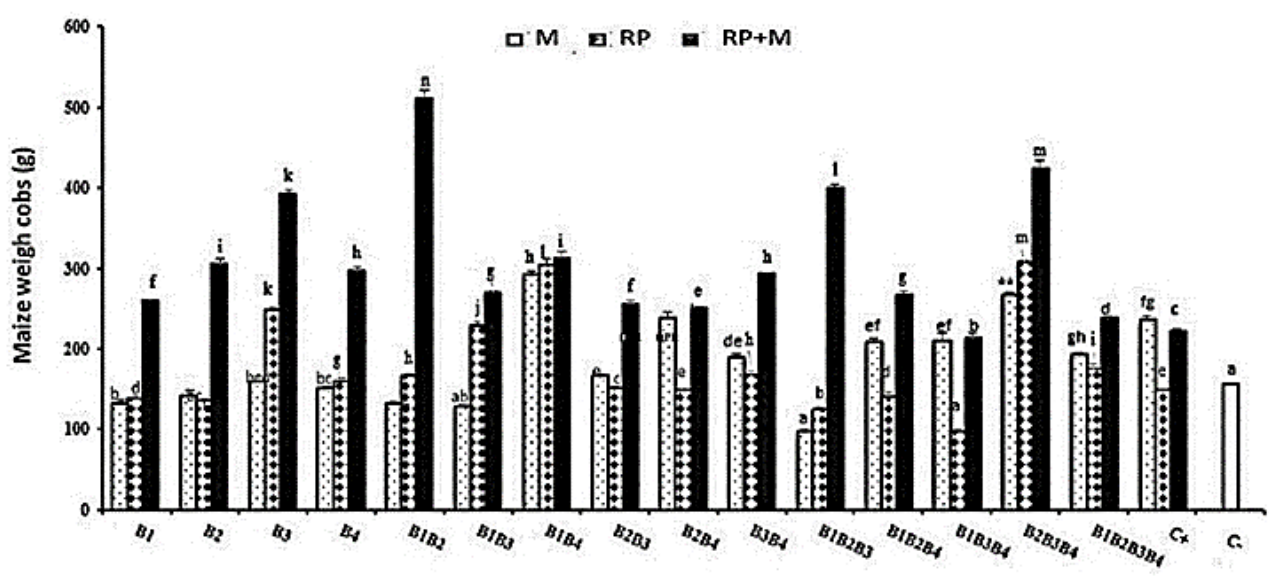

PSM Isolates

Fig. 5. Impact of Mycorrhiza, PSM and Rock powder on the weight of Maize cobs.

Legend: M: Mycorrhizal fungi, RP: Rock Powder (vivianite), RP+M: Mixing Rock Powder and Mycorrhizal fungi, C+: Positive control; C-: Negative Control with B1: BGL12, B2: SSL9, B3: BNBL17, B4: RBNBL5 as different PSM isolates.

The values with different letters are significantly different.

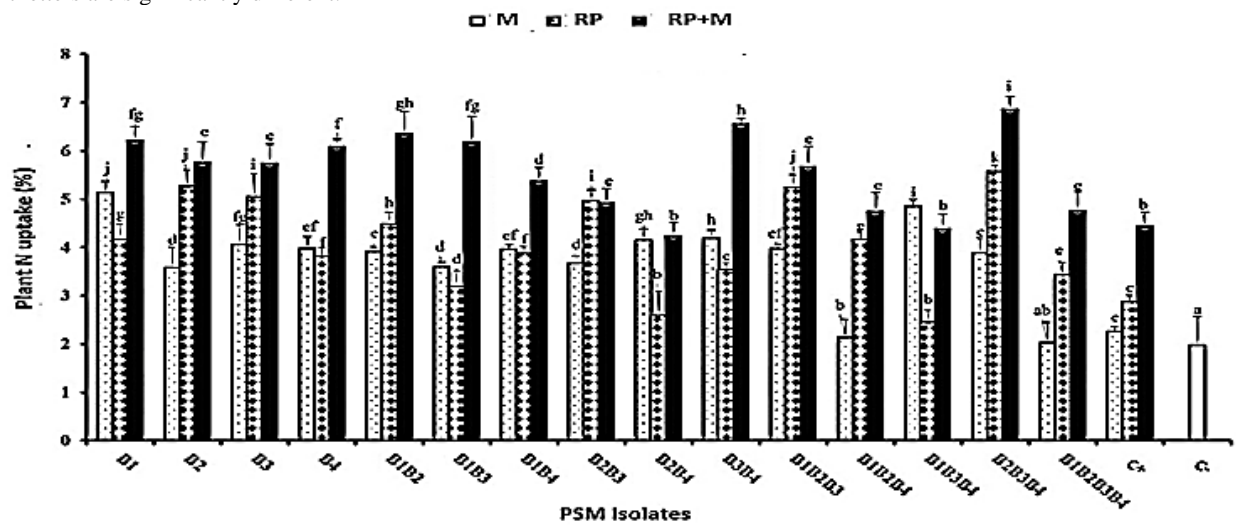

Fig. 6. Impact of treatments and PSM on nitrogen content in maize plants.

Legend: M: Mycorrhizal fungi, RP: Rock Powder (vivianite), RP+M: Mixing Rock Powder and Mycorrhizal fungi, C+: Positive control; C-: Negative Control with B1: BGL12, B2: SSL9, B3: BNBL17, B4: RBNBL5 as different PSM isolates.

The values with different letters are significantly different. 
TABLE II: EFFECTS OF DIFFERENT TREATMENTS ON TOTAL NITROGEN UPTAKE OF MAIZE PLANTS

\begin{tabular}{|c|c|c|c|c|c|c|c|c|c|c|c|c|}
\hline \multirow[t]{2}{*}{ Treatments } & \multicolumn{4}{|c|}{$\mathbf{M}$} & \multicolumn{4}{|c|}{$\mathbf{R P}$} & \multicolumn{4}{|c|}{$\mathbf{R P}+\mathbf{M}$} \\
\hline & GY & TNU & TNY* & $(\%) * *$ & GY & TNU & TNY* & $(\%) * *$ & GY & TNU & TNY* & $(\%)$ \\
\hline B1 & $1.49 \pm 0.05 \mathbf{c}$ & $7.70 \pm 0.51 \mathrm{de}$ & $107.41 \pm 5.66 \mathrm{e}$ & $190.53 \pm 4.33 \mathbf{e}$ & $1.58 \pm 0.08 \mathrm{e}$ & $5.62 \pm 0.047 \mathrm{c}$ & $88.86 \pm 7.51 \mathbf{c}$ & $140.36 \pm 1.80 \mathrm{~d}$ & $.75 \pm 0.09 d$ & $9.97 \pm 0.44 \mathbf{d}$ & $325.10 \pm 3.10 \mathrm{~d}$ & $779.36 \pm 1.96 \mathrm{e}$ \\
\hline B2 & $1.64 \pm 0.04 \mathbf{e}$ & $8.45 \pm 1.13 \mathbf{e}$ & $145.16 \pm 7.91 \mathrm{~g}$ & $292.61 \pm 3.96 \mathbf{g}$ & $1.72 \pm 0.04 \mathbf{g}$ & $11.90 \pm 0.56 \mathbf{m}$ & $204.70 \pm 9.63 \mathrm{~g}$ & $453.70 \pm 3.60 \mathrm{~h}$ & $3.33 \pm 0.00 \mathrm{~g}$ & $11.45 \pm 0.55 \mathbf{h}$ & $426.90 \pm 6.07 \mathbf{e}$ & $1054.72 \pm 1.96 \mathbf{f}$ \\
\hline B3 & $2.10 \pm 0.03 \mathrm{j}$ & $7.15 \pm 1.30 \mathrm{de}$ & $160.76 \pm 9.69 \mathbf{h}$ & $334.83 \pm 2.84 \mathbf{h}$ & $2.09 \pm 0.09 \mathbf{k}$ & $10.76 \pm 0.671$ & $224.70 \pm 14.00 \mathbf{h}$ & $507.80 \pm 3.60 \mathbf{i}$ & $4.46 \pm 0.031$ & $11.68 \pm 0.46 \mathbf{h i}$ & $489.30 \pm 1.90 \mathbf{h}$ & $1223.50 \pm 1.00 \mathbf{g l}$ \\
\hline B4 & $1.44 \pm 0.05 \mathbf{b}$ & $6.39 \pm 0.97 \mathbf{d e}$ & $83.45 \pm 1.16 \mathrm{c}$ & $125.72 \pm 8.80 \mathbf{c}$ & $1.55 \pm 0.09 \mathbf{e}$ & $7.11 \pm 0.42 \mathrm{fg}$ & $110.33 \pm 6.63 \mathbf{d}$ & $198.43 \pm 1.05 \mathbf{e}$ & $3.74 \pm 0.02 \mathbf{i}$ & $11.14 \pm 0.36 \mathbf{f g}$ & $525.72 \pm 2.90 \mathbf{i j}$ & $1327.42 \pm 1.00 \mathbf{h}$ \\
\hline B1B2 & $1.60 \pm 0.01 \mathrm{~d}$ & $7.74 \pm 0.042 \mathbf{d e}$ & $119.83 \pm 0.98 \mathbf{f}$ & $224.13 \pm 1.60 \mathbf{f}$ & $1.44 \pm 0.05 \mathbf{c}$ & $7.81 \pm 0.34 \mathrm{hi}$ & $112.80 \pm 4.99 \mathrm{~d}$ & $205.11 \pm 1.66 \mathbf{e}$ & $5.71 \pm 0.01 \mathbf{e}$ & $13.36 \pm 0.55 \mathbf{j}$ & $570.75 \pm 1.98 \mathbf{j}$ & $1443.81 \pm 2.80 \mathbf{i}$ \\
\hline B1B3 & $1.40 \pm 0.03 \mathbf{a}$ & $7.52 \pm 1.33 \mathrm{de}$ & $115.84 \pm 3.20$ ef & $231.33 \pm 1.96 \mathbf{f}$ & $2.21 \pm 0.03 \mathbf{m}$ & $7.66 \pm 0.56 \mathrm{ghi}$ & $169.40 \pm 12.40 \mathbf{f}$ & $358.21 \pm 2.20 \mathrm{~g}$ & $3.2 \pm 0.00 \mathbf{f}$ & $11.64 \pm 0.65 \mathbf{h i}$ & $411.66 \pm 4.90 \mathbf{e}$ & $1013.50 \pm 1.50 \mathbf{f}$ \\
\hline B1B4 & $1.97 \pm 0.06 \mathbf{g h}$ & $5.93 \pm 0.67 \mathbf{c d}$ & $122.33 \pm 3.80 \mathbf{f}$ & $230.88 \pm 1.08 \mathbf{f}$ & $2.35 \pm 0.07 \mathbf{n}$ & $8.63 \pm 0.46 \mathbf{i}$ & $202.60 \pm 9.94 \mathrm{~g}$ & $448.12 \pm 1.95 \mathbf{h}$ & $3.95 \pm 0.02 \mathbf{k}$ & $12.16 \pm 0.33 \mathbf{i}$ & $421.60 \pm 3.70 \mathbf{e}$ & $1040.38 \pm 1.03 \mathbf{f}$ \\
\hline B2B3 & $2.07 \pm 0.04 \mathbf{i}$ & $6.50 \pm 0.20 \mathrm{de}$ & $108.43 \pm 1.50 \mathbf{e}$ & $193.29 \pm 1.35 \mathbf{e}$ & $1.87 \pm 0.05 \mathbf{j}$ & $9.62 \pm 0.51 \mathbf{k}$ & $179.60 \pm 9.66 \mathbf{f}$ & $385.80 \pm 2.32 \mathbf{g}$ & $3.10 \pm 0.02 \mathbf{e}$ & $9.64 \pm 0.31 \mathbf{d}$ & $290.96 \pm 2.41 \mathrm{~d}$ & $687.01 \pm 1.70 \mathbf{d}$ \\
\hline B2B4 & $1.76 \pm 0.07 \mathbf{f}$ & $8.34 \pm 1.90 \mathbf{e}$ & $163.90 \pm 5.40 \mathbf{h}$ & $343.33 \pm 9.32 \mathbf{h i}$ & $1.80 \pm 0.02 \mathbf{i}$ & $7.53 \pm 0.46 \mathrm{gh}$ & $135.40 \pm 8.36 \mathbf{e}$ & $266.24 \pm 3.50 \mathbf{f}$ & $3.31 \pm 0.07 \mathbf{g}$ & $8.50 \pm 0.47 \mathbf{c}$ & $336.98 \pm 5.40 \mathbf{d}$ & $811.43 \pm 9.05 \mathbf{e}$ \\
\hline B3B4 & $1.51 \pm 0.09 \mathrm{c}$ & $6.52 \pm 0.04 \mathrm{de}$ & $96.00 \pm 3.21 \mathrm{~d}$ & $159.67 \pm 7.12 \mathrm{~d}$ & $2.14 \pm 0.05 \mathbf{I}$ & $6.34 \pm 0.29 \mathrm{de}$ & $135.80 \pm 6.14 \mathbf{e}$ & $267.32 \pm 1.75 \mathbf{f}$ & $3.89 \pm 0.01 \mathbf{j}$ & $11.21 \pm 0.11 \mathbf{f g}$ & $460.77 \pm 3.86 \mathbf{f g}$ & $1146.33 \pm 2.55 \mathrm{~g}$ \\
\hline B1B2B3 & $2.30 \pm 0.03 \mathbf{l}$ & $5.91 \pm 1.47 \mathbf{c d}$ & $154.24 \pm 2.56 \mathrm{gh}$ & $317.20 \pm 1.63 \mathbf{g h}$ & $1.64 \pm 0.04 \mathbf{f}$ & $8.31 \pm 0.44 \mathbf{i j}$ & $136.10 \pm 7.31 \mathbf{e}$ & $268.14 \pm 1.52 \mathrm{f}$ & $5.25 \pm 0.07 \mathbf{n}$ & $10.16 \pm 0.47 \mathbf{d e}$ & $480.56 \pm 3.42 \mathbf{g h}$ & $1199.86 \pm 3.83 \mathrm{~g}$ \\
\hline B1B3B4 & $2.50 \pm 0.07 \mathbf{m}$ & $7.80 \pm 0.44 \mathrm{de}$ & $202.89 \pm 8.10 \mathbf{j}$ & $448.80 \pm 8.73 \mathbf{k}$ & $1.18 \pm 0.04 \mathbf{a}$ & $5.89 \pm 0.37 \mathbf{c d}$ & $69.28 \pm 4.32 \mathbf{b}$ & $87.40 \pm 2.83 \mathbf{b c}$ & $2.63 \pm 0.05 \mathbf{b}$ & $10.73 \pm 0.37$ ef & $433.70 \pm 6.70 \mathbf{f}$ & $1073.11 \pm 1.60 f$ \\
\hline B2B3B4 & $2.27 \pm 0.06 \mathbf{k}$ & $6.63 \pm 0.40 \mathrm{de}$ & $176.75 \pm 9.35 \mathbf{i}$ & $378.10 \pm 7.93 \mathbf{j}$ & $2.83 \pm 0.00 \mathbf{o}$ & $11.33 \pm 0.01 \mathbf{m}$ & $321.30 \pm 3.20 \mathbf{i}$ & $769.08 \pm 2.80 \mathbf{j}$ & $5.17 \pm 0.01 \mathrm{~m}$ & $14.37 \pm 0.30 \mathbf{k}$ & $518.97 \pm 2.50 \mathbf{i}$ & $1303.76 \pm 2.67 \mathbf{h}$ \\
\hline B1B2B3B4 & $1.43 \pm 0.02 \mathbf{a b}$ & $4.20 \pm 0.80 \mathbf{b c}$ & $62.38 \pm 7.10 \mathbf{b}$ & $68.73 \pm 9.90 \mathbf{b}$ & $1.40 \pm 0.05 \mathbf{b}$ & $6.74 \pm 0.25$ ef & $94.71 \pm 3.63 \mathbf{c}$ & $156.18 \pm 1.14 d$ & $2.64 \pm 0.06 \mathbf{b}$ & $10.11 \pm 0.44 \mathbf{d e}$ & $237.61 \pm 2.41 \mathbf{c}$ & $542.71 \pm 3.85 \mathrm{c}$ \\
\hline $\mathrm{C}+$ & $1.95 \pm 0.04 \mathbf{g}$ & $3.17 \pm 0.56 \mathbf{a b}$ & $66.82 \pm 3.24 \mathbf{b}$ & $80.74 \pm 3.27 \mathbf{b}$ & $1.50 \pm 0.02 \mathrm{~d}$ & $4.28 \pm 0.09 \mathbf{b}$ & $64.31 \pm 1.50 \mathbf{b}$ & $73.95 \pm 1.22 \mathbf{b}$ & $2.80 \pm 0.08 \mathbf{c}$ & $6.33 \pm 0.40 \mathbf{b}$ & $134.81 \pm 5.17 \mathbf{b}$ & $264.64 \pm 35.95 \mathbf{b}$ \\
\hline C- & $1.45 \pm 0.02 \mathbf{b}$ & $2.55 \pm 0.16 \mathbf{a}$ & $36.97 \pm 2.13 \mathbf{a}$ & $0.00 \pm 0.00 \mathbf{a}$ & $1.45 \pm 0.02 \mathbf{c}$ & $2.55 \pm 0.16 \mathbf{a}$ & $36.97 \pm 2.13 \mathbf{a}$ & $0.00 \pm 0.00 \mathbf{a}$ & $1.45 \pm 0.02 \mathbf{a}$ & $2.55 \pm 0.16 \mathbf{a}$ & $36.97 \pm 2.13 \mathbf{a}$ & $0.00 \pm 0.00 \mathbf{a}$ \\
\hline Average & $1.83 \pm 0.36$ & $6.85 \pm 1.13$ & $127.76 \pm 38.41$ & $246.76 \pm 103.61$ & $1.84 \pm 0.43$ & $8.11 \pm 1.99$ & $153.14 \pm 65.54$ & $314.23 \pm 177.30$ & $3.85 \pm 0.93$ & $11.08 \pm 1.48$ & $417.80 \pm 95.20$ & $1030.45 \pm 257.94$ \\
\hline
\end{tabular}

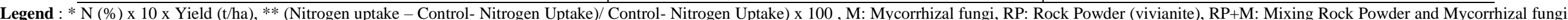
GY: Grain Yield (t/ha), TNU: Total Nitrogen Uptake (g/100g), TNY: Total Nitrogen Yield (Kg/ha), C+: Positive control, C-: Negative Control, B1: BGL12 ; B2: SSL9, B3: BNBL17; B4: RBNBL5.

The values with different letters are significantly different. 
TABLE III: EFFECTS OF DIFFERENT TREATMENTS ON TOTAL PHOSPHORUS UPTAKE OF MAIZE PLANTS

\begin{tabular}{|c|c|c|c|c|c|c|c|c|c|c|c|c|}
\hline \multirow[t]{2}{*}{ Treatments } & \multicolumn{4}{|c|}{ M } & \multicolumn{4}{|c|}{$\mathbf{R P}$} & \multicolumn{4}{|c|}{$\mathbf{R P}+\mathbf{M}$} \\
\hline & GY & TPU & TPY* & $(\%)^{* *}$ & GY & TPU & TPY* & $(\%)^{* *}$ & GY & TPU & TPY* & $(\%)^{* *}$ \\
\hline B1 & $1.49 \pm 0.05 \mathbf{c}$ & $0.57 \pm 0.02 \mathrm{gh}$ & $8.57 \pm 0.06 \mathbf{i}$ & $248.37 \pm 1.20 \mathbf{h}$ & $1.58 \pm 0.08 \mathbf{e}$ & $0.38 \pm 0.08 \mathbf{d e f g}$ & $6.11 \pm 1.36 \mathrm{e}$ & $148.37 \pm 8.4$ de & $3.75 \pm 0.09 \mathbf{d}$ & $0.51 \pm 0.14 \mathbf{c}$ & $16.5 \pm 0.23 \mathbf{b}$ & $570.73 \pm 1.90 \mathbf{d}$ \\
\hline B2 & $1.64 \pm 0.04 \mathrm{e}$ & $0.42 \pm 0.02 \mathbf{e}$ & $7.04 \pm 0.04 \mathbf{f}$ & $186.17 \pm 1.90 \mathbf{e}$ & $1.72 \pm 0.04 \mathrm{~g}$ & $0.34 \pm 0.06$ cdef & $5.95 \pm 1.15 \mathbf{d}$ & $141.86 \pm 1.62 \mathrm{de}$ & $3.33 \pm 0.00 \mathrm{~g}$ & $0.60 \pm 0.03 \mathbf{c d}$ & $20.15 \pm 0.75 \mathbf{c d}$ & $719.10 \pm 1.03 \mathbf{f}$ \\
\hline B3 & $2.10 \pm 0.03 \mathbf{j}$ & $0.33 \pm 0.01 \mathbf{c d}$ & $6.97 \pm 0.03 \mathbf{f}$ & $183.33 \pm 2.24 \mathbf{d e}$ & $2.09 \pm 0.09 \mathbf{k}$ & $0.32 \pm 0.04$ cde & $6.74 \pm 0.94$ ef & $173.98 \pm 5.52 \mathrm{f}$ & $4.46 \pm 0.031$ & $0.64 \pm 0.00 \mathrm{~d}$ & $30.61 \pm 0.46 \mathbf{g h}$ & $1144.30 \pm 1.30 \mathbf{I}$ \\
\hline B4 & $1.44 \pm 0.05 \mathbf{b}$ & $0.46 \pm 0.01$ ef & $6.70 \pm 0.03 \mathbf{e}$ & $172.35 \pm 1.70 \mathbf{d}$ & $1.55 \pm 0.09 \mathbf{e}$ & $0.46 \pm 0.08 \mathrm{~g}$ & $7.16 \pm 1.34 \mathbf{f}$ & $191.05 \pm 1.86 \mathbf{g}$ & $3.74 \pm 0.02 \mathbf{i}$ & $0.78 \pm 0.02 \mathrm{de}$ & $28.6 \pm 1.38 \mathrm{~g}$ & $1062.60 \pm 4.5 \mathbf{k}$ \\
\hline B1B2 & $1.6 \pm 0.01 \mathrm{~d}$ & $0.37 \pm 0.01 \mathbf{d}$ & $5.48 \pm 0.06 \mathbf{d}$ & $122.76 \pm 4.2 \mathrm{c}$ & $1.44 \pm 0.05 \mathbf{c}$ & $0.42 \pm 0.08$ efg & $6.11 \pm 1.14 \mathbf{e}$ & $148.37 \pm 1.01$ de & $5.71 \pm 0.01 \mathbf{e}$ & $0.75 \pm 0.05$ de & $42.13 \pm 1.75 \mathbf{j}$ & $1612.60 \pm 2.10 \mathrm{o}$ \\
\hline B1B3 & $1.4 \pm 0.03 \mathbf{a}$ & $0.48 \pm 0.06 \mathbf{f}$ & $7.2 \pm 0.06 \mathbf{f}$ & $192.68 \pm 1.95 \mathrm{e}$ & $2.21 \pm 0.03 \mathbf{m}$ & $0.44 \pm 0.06 \mathbf{f g}$ & $9.87 \pm 1.27 \mathbf{h}$ & $301.21 \pm 1.22 \mathbf{i}$ & $3.2 \pm 0.00 \mathbf{f}$ & $0.71 \pm 0.17 \mathbf{d e}$ & $21.94 \pm 0.31 \mathbf{d}$ & $791.86 \pm 3.70 \mathrm{~g}$ \\
\hline B1B4 & $1.97 \pm 0.06 \mathbf{g h}$ & $0.25 \pm 0.06 \mathbf{b}$ & $5.04 \pm 0.06 \mathbf{c}$ & $104.87 \pm 1.6 \mathbf{b}$ & $2.35 \pm 0.07 \mathbf{n}$ & $0.34 \pm 0.08$ cde & $7.94 \pm 1.83 \mathrm{~g}$ & $222.76 \pm 4.50 \mathbf{h}$ & $3.95 \pm 0.02 \mathbf{k}$ & $0.58 \pm 0.03 \mathbf{c}$ & $23.78 \pm 2.01$ ef & $866.66 \pm 2.90 \mathbf{i}$ \\
\hline B2B3 & $2.07 \pm 0.04 \mathbf{i}$ & $0.37 \pm 0.03 \mathbf{c d}$ & $7.74 \pm 0.20 \mathrm{~g}$ & $214.63 \pm 1.70 \mathbf{f}$ & $1.87 \pm 0.05 \mathbf{j}$ & $0.33 \pm 0.06$ cde & $6.20 \pm 1.17 \mathbf{e}$ & $152.03 \pm 1.82 \mathrm{e}$ & $3.1 \pm 0.02 \mathbf{e}$ & $0.66 \pm 0.01 \mathrm{~d}$ & $19.88 \pm 0.92 \mathbf{c}$ & $708.13 \pm 3.80$ ef \\
\hline B2B4 & $1.76 \pm 0.07 \mathbf{f}$ & $0.54 \pm 0.02 \mathrm{~g}$ & $9.26 \pm 0.36 \mathbf{j}$ & $276.42 \pm 2.20 \mathbf{i}$ & $1.8 \pm 0.02 \mathbf{i}$ & $0.41 \pm 0.06 \mathrm{efg}$ & $7.37 \pm 1.16 \mathbf{f}$ & $199.59 \pm 1.56 \mathbf{g}$ & $3.31 \pm 0.07 \mathbf{g}$ & $0.60 \pm 0.03 \mathbf{c d}$ & $22.85 \pm 2.40 \mathbf{e}$ & $828.86 \pm 6.30 \mathbf{h}$ \\
\hline B3B4 & $1.51 \pm 0.09 \mathrm{c}$ & $0.37 \pm 0.03 \mathbf{d}$ & $5.63 \pm 0.23 \mathbf{d}$ & $128.86 \pm 2.12 \mathbf{c}$ & $2.14 \pm 0.05 \mathbf{l}$ & $0.33 \pm 0.05$ cde & $7.16 \pm 1.14 \mathbf{f}$ & $191.05 \pm 1.40 \mathrm{~g}$ & $3.89 \pm 0.01 \mathbf{j}$ & $0.85 \pm 0.10 \mathbf{f}$ & $31.02 \pm 1.09 \mathbf{h}$ & $1160.97 \pm 2.50 \mathrm{I}$ \\
\hline B1B2B3 & $2.3 \pm 0.03 \mathbf{l}$ & $0.35 \pm 0.00 \mathbf{c d}$ & $8.20 \pm 0.15 \mathbf{h}$ & $233.33 \pm 2.31 \mathrm{~g}$ & $1.64 \pm 0.04 \mathbf{f}$ & $0.26 \pm 0.05 \mathbf{b c}$ & $4.32 \pm 0.80 \mathbf{c}$ & $75.60 \pm 2.08 \mathbf{c}$ & $5.25 \pm 0.07 \mathbf{n}$ & $0.68 \pm 0.04 \mathbf{d}$ & $35.48 \pm 1.04 \mathbf{i}$ & $1342.27 \pm 1.90 \mathrm{~m}$ \\
\hline B1B2B4 & $1.99 \pm 0.05 \mathbf{h}$ & $0.43 \pm 0.03$ ef & $8.64 \pm 0.40 \mathbf{i}$ & $251.22 \pm 1.02 \mathbf{h}$ & $1.76 \pm 0.04 \mathbf{h}$ & $0.33 \pm 0.06$ cde & $5.82 \pm 1.12 \mathbf{d}$ & $136.58 \pm 1.72 \mathbf{d}$ & $3.60 \pm 0.04 \mathbf{h}$ & $0.69 \pm 0.09 \mathbf{d}$ & $24.46 \pm 1.80 \mathbf{f}$ & $894.30 \pm 2.70 \mathbf{j}$ \\
\hline B1B3B4 & $2.57 \pm 0.07 \mathbf{m}$ & $0.60 \pm 0.01 \mathbf{h}$ & $15.7 \pm 0.01 \mathrm{I}$ & $538.21 \pm 1.80 \mathbf{k}$ & $1.18 \pm 0.04 \mathbf{a}$ & $0.35 \pm 0.07$ cdef & $4.11 \pm 0.82 \mathbf{c}$ & $67.07 \pm 1.61 \mathrm{c}$ & $2.63 \pm 0.05 \mathbf{b}$ & $0.66 \pm 0.03 \mathbf{d}$ & $19.56 \pm 1.17 \mathbf{c}$ & $695.12 \pm 1.40 \mathbf{e}$ \\
\hline B2B3B4 & $2.27 \pm 0.06 \mathbf{k}$ & $0.43 \pm 0.00$ ef & $9.93 \pm 0.06 \mathbf{k}$ & $303.66 \pm 1.08 \mathbf{j}$ & $2.83 \pm 0.00 \mathbf{o}$ & $0.34 \pm 0.05$ cde & $9.60 \pm 1.62 \mathbf{h}$ & $290.24 \pm 2.40 \mathrm{~g}$ & $5.17 \pm 0.01 \mathbf{m}$ & $0.90 \pm 0.20 \mathbf{f}$ & $41.04 \pm 1.23 \mathbf{j}$ & $1568.29 \pm 1.74 \mathbf{n}$ \\
\hline В1B2B3B4 & $1.43 \pm 0.02 \mathbf{a b}$ & $0.32 \pm 0.00 \mathbf{c}$ & $4.63 \pm 0.11 \mathbf{b}$ & $88.21 \pm 1.89 \mathbf{b}$ & $1.4 \pm 0.05 \mathbf{b}$ & $0.36 \pm 0.05 \mathrm{~cd}$ & $4.16 \pm 0.71 \mathrm{c}$ & $69.10 \pm 1.33 \mathbf{c}$ & $2.64 \pm 0.06 \mathbf{b}$ & $0.55 \pm 0.00 \mathbf{c}$ & $15.84 \pm 0.42 \mathbf{b}$ & $543.90 \pm 2.87 \mathbf{c}$ \\
\hline $\mathrm{C}+$ & $1.95 \pm 0.04 \mathrm{~g}$ & $0.23 \pm 0.01 \mathbf{b}$ & $4.50 \pm 0.18 \mathbf{b}$ & $82.93 \pm 2.14 \mathbf{b}$ & $1.5 \pm 0.02 \mathrm{~d}$ & $0.17 \pm 0.03 \mathbf{a b}$ & $2.60 \pm 0.50 \mathbf{b}$ & $5.69 \pm 1.20 \mathbf{b}$ & $2.8 \pm 0.08 \mathbf{c}$ & $0.35 \pm 0.09 \mathbf{b}$ & $15.48 \pm 0.80 \mathbf{b}$ & $529.26 \pm 2.40 \mathbf{b}$ \\
\hline C- & $1.45 \pm 0.02 \mathbf{b}$ & $0.17 \pm 0.00 \mathbf{a}$ & $1.30 \pm 0.00 \mathbf{a}$ & $0.00 \pm 0.00 \mathbf{a}$ & $1.45 \pm 0.02 \mathbf{c}$ & $0.17 \pm 0.00 \mathbf{a}$ & $1.30 \pm 0.00 \mathbf{a}$ & $0.00 \pm 0.00 \mathbf{a}$ & $1.45 \pm 0.02 \mathbf{a}$ & $0.17 \pm 0.06 \mathbf{a}$ & $1.30 \pm 0.00 \mathbf{a}$ & $0.00 \pm 0.00 \mathbf{a}$ \\
\hline Average & $1.83 \pm 0.37$ & $0.42 \pm 0.10$ & $7.78 \pm 2.69$ & $216.34 \pm 109.26$ & $1.84 \pm 0.43$ & $0.36 \pm 0.05$ & $6.57 \pm 1.73$ & $167.26 \pm 70.19$ & $3.85 \pm 0.93$ & $0.68 \pm 0.11$ & $26.26 \pm 8.32$ & $967.31 \pm 338.16$ \\
\hline
\end{tabular}

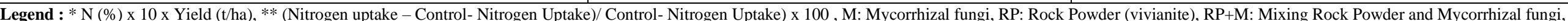
GY: Grain Yield (t/ha), TNU: Total Nitrogen Uptake (g/100g), TNY: Total Nitrogen Yield (Kg/ha), C+: Positive control, C-: Negative Control, B1: BGL12; B2: SSL9, B3: BNBL17; B4: RBNBL5.

The values with different letters are significantly different. 


\section{DISCUSSION}

In our field experiment, PSM isolates, Rocks Phosphates and Mycorrhiza associated or not were used to evaluate some parameters as maize cobs, nitrogen uptake, phosphorus uptake and grain yield. The performance of the four isolates in association or alone (B1: BGL12, B2: SSL9, B3: BNBL17 and B4: RBNBL17) in increasing these parameters showed that they might have mineralized the rocks phosphate (RP) by releasing phosphatase enzyme, organic or inorganics acids. The results are similar to that reported by [4], [27], [6], [7], [18]. A highly significant difference $(\mathrm{P}<0.001)$ was observed between the effects of PSM isolates and the average weight of maize cobs for the $\mathrm{RP}+\mathrm{M}$ treatment. The effect of $\mathrm{RP}+\mathrm{M}$ treatment associated with isolates promoted nitrogen content than with the RP and M treatments alone. The average nitrogen content of plants (B2B3B4: $6.85 \pm 0.15$ $\mathrm{mg} / 100 \mathrm{~g}$ ) in the $\mathrm{RP}+\mathrm{M}$ treatment improved nitrogen yield (B1B2: $570.75 \pm 1.98 \mathrm{Kg} / \mathrm{ha}$ ) and the percentage of nitrogen absorption (B1B2: $1443.42 \pm 2.80 \%$ ) of this element compared to the negative control (C-). However, the RP treatment without PSM isolates has no effect on these two parameters, this indicate the importance of PSM and Mycorrhiza. The analysis of variance showed that a significant difference was observed between the effects of PSM isolates and the phosphorus mean yield content (B2B3B4: $41.04 \pm 1.23 \mathrm{Kg} / \mathrm{ha}$ ) and the percentage of phosphorus absorption (B2B3B4: 1568.29 $\pm 1.74 \%$ ) for $\mathrm{RP}+\mathrm{M}$ treatment. Many authors showed the role of Phosphate Solubilizing Microorganisms (PSM) in releasing P from rock phosphates [11], [25], [17], [1], [8], [34], [26] which may lead to increased P uptake and plant growth [12]. Co-inoculation of PSM, Arbuscular Mycorrhizal fungi (AMF) with Rocks Phosphates resulted in significantly higher of nitrogen and phosphorus uptake in plant than where these microorganisms were used alone. For all the inoculation effect, co-inoculation with the AMF and PSM resulted in the highest plant growth response. Some reports of field trials have showed the beneficial effects of co-inoculations with Phosphate Solubilizing Bacteria (PSB) and AMF [13], [25] and PSM survive longer when associated to Mycorrhizal roots rather than to non-Mycorrhizal roots [30], [2], thus increasing the possibility of delivering $\mathrm{P}$ into the soil solution.

\section{CONCLUSION}

The objective of this work was to evaluate the effect of PSM associated with RP and Mycorrhizae on Zea mays growth. Results indicate that these isolates can effectively improve the solubility of $\mathrm{P}$ content in rock phosphate applied as fertilizer, thus increase the amount of available nitrogen and phosphorus in the soil and maize productivity. Also results showed that solubilization or RP was better with double inoculation of PSM and Mycorrhiza. PSM and RP can be used as natural fertilizers to improve plants productivity in the Northern Cameroon.

\section{ACKNOWLEDGEMENTS}

The authors are thankful to Mr Mohfenka Peter who corrected this article. This research did not receive any specific grant from funding agencies in the public, commercial or not-for-profit sectors.

\section{REFERENCES}

[1] Babana, A. H., Dicko, A. H., Maïga, K. \& Traoré, D. 2013. Characterization of rock phosphate solubilizing microorganisms isolated from wheat (Triticum aestivum L.) rhizosphere in Mali. Journal of Microbiology and Microbial Research 1: pp.1-6.

[2] Barea, J.M., Azco'n, R., Azco'n-Aguilar C. 2002. Mycorrhizosphere interactions to improve plant fitness and soil quality. Antonie Van Leeuwenhoek 81:343-351.

[3] Cairns, J. E., Hellin, J., Sonder, K. et al., 2013. "Adapting maize production to climate change in sub-Saharan Africa," Food Security 5: 345-360.

[4] Chabot, R. Antoun, H. \& Cescas, MP. 1996. Growth promotion of maize and lettuce by phosphate solubilizing Rhizobium leguminosarum biovar. phaseoli. Plant Soil 184:311-321.

[5] Devani, M.B., Shishoo, J.C., Shal, S.A. \& Suhagia, B.N. 1989. Spectrophotometrical method for micro determination of nitrogen in Kjeldahl digest. Journal Association Off. Analatycal Chemistry 72: 953-956.

[6] El-Tarabily, K.A., Nassar, A.H. and Sivasithamparam, K. 2008. Promotion of growth of bean (Phaseolus vulgaris L.) in a calcareous soil by a phosphate-solubilizing, rhizospherecompetent isolate of Micromonospora endolithica. Applied Soil Ecology 39:161-171.

[7] Fankem, H., Ngo Nkot, L., Deubel, A., Quinn, J., Merbach, W., Etoa, F.X. \& Nwaga, D. 2008. Solubilization of inorganic phosphates and plant growth promotion by strains of Pseudomonas fluorescens isolated from acidic soils of Cameroon. African Journal of Microbiological Research 2: 171-178.

[8] Fankem, H., Tchuisseu Tchakounté, G.V., Ngo Nkot, L., Nguesseu Njanjouo, G., Nwaga, D., Etoa, F-X. 2014. Maize (Zea mays) growth promotion by rock-phosphate solubilising bacteria isolated from nutrient deficient soils of Cameroon. African Journal of Microbiology Research 8: 3570-3579.

[9] Gouda, S., Kerry, R.G., Das, G., Paramithiotis, S., Shin, H.-S., Patra, J.K. 2018. Revitalization of plant growth promoting rhizobacteria for sustainable development in agriculture. Microbiology Research 206:131-140.

[10] Gyaneshwar, P., Naresh, K.G., Parekh, L.J. \& Poole, P.S. 2002 Role of soil microorganisms in improving $\mathrm{P}$ nutrition of plants Plant Soil 245: 83-93.

[11] Han, H.S., Supanjani, L.K.D., 2006. Effect of co-inoculation with phosphate and potassium solubilizing bacteria on mineral uptake and growth of pepper and cucumber. Plant Soil Environnement 52: 130-136.

[12] Jorquera, M.A., Hernandez, M.T., Rengel, Z., Marschner, P. \& Mora, M.L. 2008. Isolation of culturable phosphobacteria with both phytate-mineralization and phosphate-solubilization activity from the rhizosphere of plants grown in a volcanic soil. Biology and Fertility of Soils 44: 1025-1034.

[13] Khan, M. S., Zaidi, A. \& Wani, P. A. 2006. Role of phosphate solubilizing microorganisms in sustainable agriculture-A review. Agronomy Sustainable Developpment 26: 1-15.

[14] Koppelaar, R. H. E. M., \& Weikard, H. P. 2013. Assessing phosphate rock depletion and phosphorus recycling options. Global Environmental Change 23: 1454-1466. doi:10.1016/j.gloenvcha.2013.09.002.

[15] Maimouna, A., 2006. Ecologie des microorganismes du palmier à huile et caractérisation des microorganismes solubilisation le phosphore : Description, Croissance et effets sur les plantes. Mémoire de DEA, Université de Yaoundé I, 36p.

[16] Maimouna, A., Megueni C., Dieudonné, N., Fabrice, W., Bernard, A. N. \& Tanyi, K. M. 2016. Impact of selecting Phosphate Solubilizing Microorganisms on the growth of maize and sorghum using vivianite as inorganic phosphate supply. International Journal of Current Research 8: 39579-39591.

[17] Marschner, P. 2009. The role of rhizosphere microorganisms in relation to P uptake by plants. In: The Ecophysiology of PlantPhosphorus Interactions, Plant Ecophysilogy. Series (eds P.J. White \& J.P. Hammond). Springer, Heidelberg, 165-176. 
[18] Marschner, P. \& Rengel, Z. 2010. The Effects of Plant Breeding on Soil Microbes. Chapter 8. Soil Microbiology and Sustainable Crop Production. DOI 10.1007/978-90-481-9479-7_8.

[19] Megueni, C., Awono, E. T., \& Ndjouenkeu, R. 2011. Effet simultané de la dilution et de la combinaison du Rhizobium et des mycorhizes sur la production foliaire et les propriétés physicochimiques des jeunes feuilles de Vigna unguiculata (L.) Walp. In Journal of Applied Biosciences 40: 2668 - 2676.

[20] Mohamed, H. 2012. Nouvelle Alerte environnementale : épuisement du phosphore.2p.

[21] Murphy, J. \& Riley, J.P. 1962. A modified single solution method for the determination of phosphate in natural waters. Analytica Chimica Acta 27: 31-36.

[22] Ngakou, A., Tamò, M., Parh, I.A., Nwaga, D., Ntonifor, N.N. and Nebane, C.L. 2008. Management of cowpea flower thrips Megalurothrips sjostedti (Thysanoptera, Thripidae) in Cameroon. Crop Protection 27: 481-488.

[23] Nwaga, D., Hamon, S., Djieto, L C. \& Engelmann, F. 2007. Biotechnologies et maîtrise des intrants agricoles en Afrique centrale. Réseau 'BIOVEG', AUF/IRD/Université de Yaoundé I/IRAD, Yaoundé, Cameroon. http://www. bioveg.auf.org.

[24] Nwaga, D., Jansa, J., Abossolo Angue, M. et al., 2010. The potential of soil beneficial microorganisms for slash-and-burn agriculture in the humid forest zone of Sub Saharan Africa. Chap 5 In Soil Biology and Agriculture in the Tropics, P Dion (ed), SpringerVerlag, Berlin, Heidelberg, pp 81-107.

[25] Osorio, Vega, N.W. 2007. A review on beneficial effects of rhizosphere bacteria on soil nutrient availability and plant nutrient uptake. Revista Facultad Nacional de Agronomía, Medellín 60: 3621-3643.

[26] Ramatou, L. A. 2016. Résistance des microorganismes solubilisant le phosphore aux stress environnementaux et leur effet sur la croissance du maïs dans la Région de l'Adamaoua. Mémoire de Master. 57p.

[27] Richardson, A. E. 2001. Prospects for using soil microorganisms to improve the acquisition of phosphorus by plants. Australian Journal of Plant Physiology 28: 897-906.

[28] Sadeghzadeh B. A review of zinc nutrition and plant breeding. J. Soil Sci. Plant Nutr. 2013;13:905-927.

[29] Setiawati, A. \& Handayanto, E. 2010. Role of phosphate Solubilizing bacteria on availability phosphorus in Oxisols and tracing of phosphate in corn by using 32P. In: 19th World Congress of Soil Science, Soil Solutions for a Changing World: Brisbane, Australia. $4 \mathrm{p}$.

[30] Singh, H.P. 1990. Response of dual inoculation with Bradyrhizobium and VAM mycorrhiza or phosphate solubilizer on soybean in mollisol. In: Jalali BL, Chand H (eds) Trends in mycorrhizae. Research proceedings of the national conference on mycorrhiza. Hisar, India, pp 14-16.

[31] Smith, S.E. \& Read, D.J. 2008. The mycorrhizal symbiosis. San Diego, USA: Academic Press.

[32] United State Department of Agricultural. 2017. Available at: https://www.

[33] Vasconcellos, C. A., Pitta, G. V. E., Franca, G. E., and de Alves, V. M. C. 2000. Soloe Fertilizantes com fósforo. Revista Cultivar 13: $30-32$

[34] Wassouni, F. 2014. Caractérisation des microorganismes solubilisant le phosphore dans les sols de la Région de l'Adamaoua Cameroun et évaluer l'effet de leur inoculation sur la production de Zea mays et Sorghum bicolor L. Moench. Mémoire de Master, 73p.

[35] Yaya, F., Nguetnkam, J.P., Tchameni, R., Basga, S.D. \& Penaye, J. 2015. Assessment of the fertilizing effect of Vivianite on the growth and yield of the Bean "Phaseolus vulgaris" on Oxisoils from Ngaoundere (Central North Cameroon). International Research Journal of Earth Sciences, 3: 18-26.

[36] Zapata, F. \& Roy, R.N. 2004. Use of Phosphate Rock for Sustainable Agriculture. FAO and IAEA, Rome, Italy. 